\title{
La inconmensurabilidad en la era de la comunicación. Reflexiones acerca del relativismo cultural y las comunidades cerradas
}

Dante Palma*

\section{Resumen / La inconmensurabilidad en la era de la comunicación. Reflexiones acerca del relativismo cultural y las comunidades cerradas}

La problemática de la comunicación en el mundo globalizado es tema central de reflexión. A grandes rasgos el debate enfrenta una postura optimista que ve en la globalización y en las telecomunicaciones el paradigma de la civilización y de la libertad de expresión, con una postura escéptica que indica que detrás de la utopía de la comunicación sin fronteras se halla la pretensión homogeneizante de la cultura mayoritaria.

Esta última posición rescata lo particular e idiosincrásico, en la mayoría de los casos, desde una actitud relativista que afirma que las diferentes culturas son inconmensurables. La reflexión aborda teorías que vinculan el concepto de comunidad y el lenguaje en el ámbito de la reflexión política y científica.

\section{Palabras clave}

Comprensión - comunicación - comunidad - inconmensurabilidad - progreso - relativismo - traducción.

\section{Summary / Immeasurability in the age of communication. Reflections of cultural relativ- ism and closed communities}

The communication problematic in the globalized world is the central topic of relextion that goes the debate faces an optimistic stand considering the globalization and the telecommunications the a paradigm among civilization and freedom of speech, seen by a skeptical position which indicates that behind the free border communication utopia is found a homogenizing pretension of the majority culture.

This last stand values the particular and idiosyncratic, in most of the cases by a relativist posture that affirms different cultures as measureless.. The thinking over those theories that link the concept of community and language in the field of the political and scientific reflection to finish off by pointing out the difficulties that relativists theories faced to grant descriptive and normative tools explaining particular circumstances of our present.

\section{Key words}

Comprehension - communication - community - immeasurability - progress - relativism - translation.

\section{Resumo / 0 Immeasurability na idade de comunicação. Reflexão respeito do relativismo cultural e as comunidades fechadas.}

A problemática da comunicação no mundo globalizado é, com certeza, um dos temas centrais que atravessa diferentes âmbitos de reflexão.

A grandes rasgos pode-se afirmar enfrenta uma postura optimista que vê na globalização das telecomunicações o paradigma da civilização e da liberdade de expressão, com uma postura asséptica que indica que detrás da utopia da comunicação sem fronteiras se encontra a pretensão homogeneizante da cultura maioritária.

Esta última posição resgata o particular e idiossincrático, na maioria dos casos, desde uma atitude relativista que afirma que as diferentes culturas são incomensuráveis

A reflexão atinge aquelas teorias que vinculam o conceito da comunidade e a linguagem no âmbito da reflexão política e científica.

\section{Palavras clave}

Compreensão - comunicação - comunidade - incomensurabilidade - progresso - relativismo - tradução.

\footnotetext{
* Dante Palma. Profesor de Filosofía (UBA). Investigador en las áreas de Epistemología y Filosofía Práctica. Docente de la Facultad de Diseño y Comunicación en la UP, la UBA y otras universidades.

infocedyc@palermo.edu.ar
} 
La problemática de la comunicación entre comunidades en la actualidad es, seguramente, uno de los temas centrales que atraviesa diferentes ámbitos de reflexión y cuyo análisis no puede realizarse independientemente de la actitud que se adopte frente al proceso de globalización. En general, podemos encontrar dos recepciones diferentes de este fenómeno.

Por un lado, los más optimistas ven en la globalización una señal del progreso civilizatorio de la humanidad que parece desembocar en un universo común de significaciones que implica un avance respecto de las circunstancias históricas pretéritas. Impulsados por las telecomunicaciones, la utopía de una opinión pública mundial para la cual la geografía no es un límite, parece ser una meta, aunque sea a mediano plazo, alcanzable.

Por otro lado, existen también voces discordantes que ven al menos con escepticismo un proceso que, en el afán de universalizar, es interpretado como aquel que iguala eliminando las diferencias. Para estas posturas, las telecomunicaciones y particularmente Internet, lejos de ser neutrales y transformase en el "paraíso de la libre expresión" no son otra cosa que nuevas formas de imperialismo cultural a la que lleva la propia lógica del capitalismo.

Esta discusión no es privativa del mundo académico sino que sus consecuencias se manifiestan de manera dramática en el ámbito político: Tras los atentados del 11 de septiembre la brecha entre la cultura occidental y la no-occidental parece haber llegado a límites insospechados en lo que algunos definen como un verdadero "choque de civilizaciones".

En el contexto de este rico debate el concepto de comunidad ha vuelto a tener la relevancia que la hegemonía del discurso liberal durante las últimas décadas le había quitado. En este sentido, los ataques a la globalización provienen especialmente de teorías que realzan el valor de la comunidad como el espacio en el que se constituye el individuo y es a partir de esta idea que los teóricos de la comunidad encuentran un argumento atendible para sus reivindicaciones ante las culturas mayoritarias en las cuales se encuentran insertos.

Este rescate de lo particular muchas veces se apoya en una actitud relativista: Cada comunidad tiene un valor en sí de lo cual se sigue que ninguna es mejor que otra o ninguna implica un progreso respecto de otra. Este relativismo se basa en el presupuesto de que las diferentes culturas resultan incomparables 0 inconmensurables de lo cual se sigue que es imposible que la comunicación permita llegar a una mejor comprensión de aquello que se presenta como "lo otro".

La incapacidad de comprender es el argumento que se utiliza para acusar a los occidentales de etnocentristas cuando éstos interpretan prácticas abusivas contra la mujer como la ablación de clítoris, la prohibición de mostrar el cuerpo públicamente o la pena de muerte a piedrazos en caso de infidelidad, como actos de barbarismo. Frente a esta actitud se advierte a los occidentales que lo que estaría ope- rando es una evaluación de una cultura desde una cosmovisión ajena.

El debate transcurre, entonces, fundamentalmente, en el terreno de la comunicación en el sentido de que las diferentes cosmovisiones que las comunidades tienen del mundo parecen imposibles de ser transmitidas de manera tal que una comunidad ajena pueda comprenderlas. De aquí que se comience a hablar de inconmensurabilidad en el sentido de que dos comunidades no poseen un espacio objetivo desde el cual poder comunicarse, evaluarse y comprenderse. Esta dificultad de comunicación nos traslada al terreno del lenguaje, perspectiva desde la cual se desarrollará el siguiente trabajo. Para ello quisiera reflexionar sobre aquellas teorías que vinculan el concepto de comunidad y el lenguaje en dos ámbitos que, en principio, pueden parecer bastante diferentes: La reflexión política y la científica.

En general se considera que el choque de culturas transcurre más en el terreno político/social -tomado el término en un sentido amplio- que en el ámbito de la ciencia dado que este último se caracterizaría por ser objetivo y universal. Sin embargo, muchos creen que no es éste el caso y que la incomparabilidad es un producto esencial de la cognición humana en tanto las culturas determinan la manera en que los sujetos perciben el mundo.

Por ello, retomaré crítica y comparativamente lo que considero son pensadores centrales de cada una de estas áreas cuyas reflexiones se encuentran presentes en los debates de hoy día. Me refiero más precisamente a las consecuencias relativistas que se siguen del concepto de comunidad -social- desarrollado especialmente por J. G. Herder hacia el fin del siglo XVIII y el uso de comunidad -científica- que la reflexión acerca de la práctica científica viene realizando desde la aparición de La Estructura de las revoluciones científicas de T. S. Kuhn en 1962.

\section{La política y la ciencia atravesadas por el lenguaje} El giro lingüístico de la filosofía europea hacia principios del S XX reabrió una serie de interrogantes filosóficos desde una perspectiva novedosa. Desde este enfoque, el lenguaje no sólo es relevante para indagar la problemática de la comunicación sino también para la teoría del conocimiento en general e incluso para la teoría política.

El lenguaje se presenta como la condición de posibilidad para acceder a un mundo que, en el ámbito del conocimiento científico en particular y especialmente a partir de las teorizaciones del Círculo de Viena, se erige como el "Tribunal del conocimiento". En esta línea, y fiel a la influencia fundamental del "primer" Wittgenstein, la ciencia no sería otra cosa que un sistema de enunciados independientes entre sí y correspondientes cada uno de ellos a expresiones lógicas, fieles y transparentes, de un estado real de cosas. Sin embargo, algunas décadas después de la formación del Círculo, T. S. Kuhn llama la atención sobre un aspecto que parecía dejarse de soslayo, esto es, la dependencia de la observación respecto de la teoría. Este señalamiento, que se en- 
cuentra en el fondo de las disputas entre nuevos y viejos epistemólogos, se expresa, en la última versión de la teoría de Kuhn, en términos lingüísticos cuando los paradigmas son definidos como léxicos o categorías taxonómicas que permiten clasificar los fenómenos del mundo.

En la teoría política es posible remontarnos a uno de los autores que más ha sido emparentado con las corrientes nacionalistas como J. G. Herder. Este alemán contemporáneo de Kant construyó su teoría de los inconmensurables "espíritus nacionales" en función de una controvertida tesis acerca del lenguaje la cual desarrollaba la relación bicondicional entre espíritu nacional y lengua en tanto el lenguaje es aquello a través de lo cual el miembro de una comunidad accede al mundo. A continuación, entonces, me propongo comparar las tematizaciones de Herder y Kuhn en sus respectivas áreas para repensar de qué manera el sostener la tesis de comunidades cerradas nos lleva, de la mano de la inconmensurabilidad, a un relativismo que plantea grandes dificultades a la hora de comprender el fenómeno de la comunicación en la actualidad.

\section{La comunidad como límite}

- La reivindicación del particularismo en Herder

La mayoría de las teorías nacionalistas, aun las más agresivas, suelen apoyarse en la teoría de J. G. Herder. Nacido en 1744 y fallecido en 1803, este alemán es uno de los representantes más conspicuos de la revuelta romántica frente al iluminismo del siglo XVIII. Frente a los contractualistas y a los enciclopedistas franceses como Voltarie y Diderot quienes depositaban su confianza en una razón universal que carecía de fronteras y que era garante del progreso ilimitado de la humanidad, Herder oponía la reacción particularista de quien veía en aquella actitud el intento de imposición de una serie de valores foráneos. En general, podemos decir que Herder trastrocó las principales banderas del iluminismo. Por ello, a lo abstracto opuso lo concreto mientras que a la cantidad y a la razón le opuso la cualidad, la fe, el misticismo y la irracionalidad.

Según Berlin (2000), el pensamiento político herderiano puede caracterizarse de la siguiente manera: Se trata, en primer lugar, de un pensamiento "populista", entendiendo por tal la creencia en el valor de la pertenencia de un individuo a un grupo o cultura; en segundo lugar se puede entrever en Herder el expresionismo propio de los románticos, esto es, la doctrina que afirma que la actividad humana especialmente la artística- de un pueblo o un individuo expresan la personalidad de aquel/los que la realizan. Por último y en tercer lugar, se encuentra su "pluralismo" entendido no sólo como el factum de la pluralidad de comunidades o valores sino la apuesta por una inconmensurabilidad de los mismos. Sin embargo, estos elementos básicos deben comprenderse a través del vínculo estrecho de la teoría política de Herder con su teoría del lenguaje. En este sentido, y a manera de aproximación, po- demos decir que en Herder se conjuga la reivindicación de lo particular pensado en clave religiosa en el sentido de responder a un plan de la divinidad que trasciende las conciencias humanas, con una tesis que afirma que aquello que identifica a las comunidades es el espíritu nacional cuya manifestación concreta es su idioma. Se trata entonces, no tanto de un nacionalismo político - pues se está hablando de naciones y no de Estados- ni tampoco exclusivamente de un nacionalismo étnico o racial. Sería más bien un nacionalismo lingüístico. De aquí que cualquier consideración que se realice sobre Herder obviando su teoría del lenguaje es, al menos, insuficiente. Quisiera centrarme, entonces, por ahora, en el conepto de comunidad que está estrechamente ligado al de Volkgeist -espíritu del pueblo- en tanto sus límites coinciden.

Lo que hace que una comunidad sea tal es el hecho de compartir una historia, un lugar, un conjunto de valores y una religión. A su vez todos estos elementos se articulan en el marco de una lengua común.

Aquí, claramente, debemos tener en cuenta la distinción entre Estado y Nación. Según Herder, el Estado es aquella estructura jurídico-política artificial y abstracta que nada tiene que ver con los vínculos entre los hombres y cuya construcción se encuentra siempre vinculada a y en beneficio de un grupo. Frente a esto, la nación aparece como el conjunto de los hombres que tienen vínculos concretos entre sí y cuya formación responde a la naturaleza.

"M illones de hombres sobre la Tierra viven sin Estado (...). Padre, madre, marido y mujer, hijos y hermanos, amigos y hombres son las relaciones naturales que pueden otorgarnos la felicidad; todo cuanto el Estado pueda darnos será siempre artificial, pero, por desgracia, puede robarnos algo mucho más esencial: ¡A nosotros mismos!" (Herder, 1959: 256) La teoría contractualista del Estado se presenta para Herder, entonces, como una formación producto del egoísmo y el cálculo utilitarista fundado en el miedo, además de encontrarse alejado del afecto y el respeto como cualidades naturales inherentes a las relaciones entre los hombres.

“ ¡Cuántos pueblos de la Tierra no saben nada de tal Estado y viven, no obstante, más felices que tantos benefactores crucificados del Estado! (...) Es manifiesto que con la grandeza de los Estados y la mayor complejidad de su composición crece necesariamente y en gran medida el peligro de multiplicar el número de individuos desdichados. En los grandes Estados, centenares pasan hambre para que uno lleve vida de calavera y sibarita. Miles y miles son oprimidos y arrojados a la muerte para que una cabeza coronada, idiota o sabia, pueda llevar a cabo sus fantasías." (Herder, 1959: 255)

Cada comunidad (entendida como nación) es un fin en sí mismo y cualquier tematización de la historia que ubique a una comunidad como un medio para un fin determinado desobedece el plan de la providencia ${ }^{2}$. Cada comunidad tiene su propio "centro de felicidad", " atmósfera" o "ethos". Como indica Parekh (2000), Herder retoma de Leibniz la idea de mónada 
y la aplica a la comunidad. La comunidad se presenta, entonces, como algo cerrado, autosuficiente y aislado de cualquier elemento foráneo.

La humanidad conformada por los hombres concretos socializados en comunidades, tiene un origen común siendo las diferencias intercomunitarias producto del influjo de factores físicos y geográficos. De esta manera, el Volkgeist no se apoya en aspectos tales como una raza, una etnia o un color.

"Como Buffon, (Herder) estima que hay variedades de la especie humana, tipos de alteraciones que se perpetúan de generación en generación. Para Herder, las posibilidades y los límites de cada nación están determinados por la cultura, con su herramienta indispensable, la lengua, pero también por las coacciones del clima y el medio geográfico." (Olender, 2005:64)

Si a esto le sumamos la adhesión herderiana al pensamiento político típico de la antigua Grecia en el que se rescata a la comunidad como aquel elemento formador de la identidad sin el cual el individuo no puede realizarse, podremos empezar a estructurar el pensamiento de un antecedente obligado de cualquier teoría nacionalista.

A diferencia del pensamiento universalista de la llustración que escinde la razón universal de los aspectos contextuales e históricos de los sujetos, Herder, en la crítica que luego retomarán los comunitaristas de finales del S. XX, afirma que el sujeto es un todo encarnado y que cualquier análisis que se apoye en separar lo aparentemente universal de las particularidades concretas, expresa, al menos, un punto de vista sesgado.

Este respeto por las comunidades, entonces, no es parte de una suerte de ley universal moral que lo prescriba sino la consecuencia de la aparente demostración natural de la importancia que tiene para la constitución de la identidad de los individuos el hecho de pertenecer a una comunidad. ${ }^{3}$

\section{- La comunidad científica: Exclusividad y aislamiento}

La teoría del físico estadounidense devenido historiador de la ciencia, T. S. Kuhn ha marcado un punto de inflexión en el estudio de la ciencia: su Estructura de la revoluciones científicas (en adelante ERC), editado en 1962, significó un cambio abrupto respecto de las teorías precedentes. A grandes rasgos, es posible afirmar que el cambio de perspectiva que ofrece Kuhn está vinculado al acento puesto en la historia como elemento ineludible de la reflexión acerca de la ciencia.

Frente a las teorías del Círculo de Viena y los epistemólogos tradicionales quienes interpretaban que las cuestiones epistemológicas pasibles de ser analizadas se vinculaban a aquello que Reichenbach ${ }^{4}$ llamó Contexto de justificación en oposición al Contexto de descubrimiento, Kuhn brinda un punto de vista que muestra la conexión inescindible entre los factores históricos que rodean a un científico -perte- necientes al contexto de descubrimiento- y los análisis lógico formales que, hasta ese momento, se presentaban como objetivos y universales en tanto a-históricos. Con este debate de fondo, la teoría de Kuhn, además, pone en tela de juicio una serie de "verdades autoevidentes" de la Epistemología estándar. En primer lugar, critica la interpretación de los propios científicos y del sentido común que construye el estereotipo del científico como aquel hombre solitario cuya vida trasciende lo meramente contextual en pos de la verdad objetiva. Frente a estos relatos Kuhn resalta la relación comunitaria que se establece entre los científicos. En otras palabras, más allá de que las teorías cobren notoriedad en tanto descubiertas por un individuo $x$ (llámese Newton, Galileo o Darwin), la práctica científica es siempre un trabajo que se realiza dentro de una comunidad la cual, a su vez, se encuentra inserta en una comunidad social más amplia anclada históricamente con intereses, valores y necesidades concretas.

Por ello, Kuhn afirma que los científicos trabajan dentro de lo que podríamos llamar una tradición, esto es, un conjunto de teorías científicas compartidas por los pares cuyo fundamento, en la mayoría de los casos, se vincula a las afirmaciones que realizaron otros científicos en tiempos pretéritos. Así podemos decir que la astronomía medieval derribada por la revolución copernicana pertenecía a la tradición aristotélica continuada en el Siglo II d.C. por Tolomeo. Asimismo, y aquí cabe mencionar la inversión propuesta por Kuhn contra las posturas positivistas ingenuas, el norteamericano señala la importancia de la teoría en tanto determinante de la observación. Esto significa que las percepciones que realicen los sujetos en su entorno estarán determinadas por una subjetividad constituida sobre la base del idioma, la cultura, los valores y los intereses de la persona.

Ahora bien, lo que a mí me interesa rescatar en este punto es de qué manera se forma esta comunidad científica que tiene en común una tradición y, en todo caso, cuáles son los elementos que determinan los límites de una comunidad en tanto diferente de otra. Para responder a esto, puede ser útil la caracterización que realiza Kuhn de las comunidades científicas. Según éste, las comunidades se encuentran aisladas y son reticentes a los cambios estando los fundamentos de esta caracterización en los particulares elementos que comparten los miembros de una comunidad. En primer lugar, una comunidad comparte un lenguaje técnico: La utilización de términos como "átomo", "masa", " planeta" o "energía" difieren de comunidad en comunidad a lo largo del tiempo y resultan muchas veces incomprensibles para comunidades que, si bien son contemporáneas, pertenecen a otra disciplina.

El lenguaje técnico de las disciplinas se aprehende en la Universidad a lo largo de la formación del profesional. De esta manera, los estudiantes adquieren un conjunto de normas, técnicas y herramientas 
por el sólo hecho de compartir una misma institución, los mismos profesores y la misma bibliografía. Asimismo, si tenemos en cuenta que muchas de las disciplinas en las que Kuhn está pensando, las de las ciencias naturales, poseen la particularidad, al menos en Estados Unidos, de ser explicadas a través de manuales y no desde las fuentes, se puede colegir de ello que la diversidad de opiniones no es aquello que busque privilegiarse y que la uniformidad será la regla más que la excepción.

Es esta formación común la que determina los "grandes problemas" de la disciplina e incluso los caminos posibles para solucionarlos. Para pensar en un ejemplo, la cuestión de la retrogradación de los astros podía ser un problema para un astrónomo aristotélico pero no para alguien que adoptara el paradigma copernicano de una Tierra en movimiento y unos astros que giran elípticamente a diferentes velocidades y con trayectorias también diferentes.

"La educación científica inculca lo que la comunidad científica conquistó anteriormente con dificultad: una profunda adhesión a un modo particular de contemplar el mundo y de practicar la ciencia en él. ( ... ) Al definir para el científico los problemas que es menester investigar y el carácter de las soluciones aceptables para ellos, tal adhesión es realmente constitutiva de la investigación. Normalmente, el científico se dedica a resolver problemas, como el jugador de ajedrez, y la adhesión que induce la educación recibida es lo que le proporciona las reglas del juego que se juega en su época." (Kuhn, 1963:349)

Pero existe un nuevo elemento que debemos tener en cuenta y que resulta controvertido. Me refiero al carácter de exclusividad de la tradición en el sentido de que la formación del científico se realiza dentro de una y sólo una tradición.

De una manera más técnica, Kuhn identificará a esto que hasta aquí hemos denominado tradición, como un paradigma siendo ésta una noción clave sobre la se ha de regresar en los próximos apartados. Sin embargo, cabe adelantar lo siguiente: A diferencia de lo que podía ocurrir en la literatura, los científicos de las ciencias duras deben tomar partido necesariamente por un paradigma. En otras palabras, mientras que es perfectamente factible que un amante de la literatura se considere devoto de estilos tan diversos como el de Borges, Galeano, Tolstoi o Shakespeare, ningún científico respetable podrá considerarse geocentrista y heliocentrista, o evolucionista y fijista simultáneamente.

Esta caracterización de las comunidades científicas como entidades cerradas, aisladas y reticentes a los cambios se asemeja bastante a la caracterización que hiciese Herder de las comunidades sociales, si bien, por supuesto, el elemento aglutinador de la comunidad científica no es ningún "espíritu del pueblo" . Sin embargo, sin metafísica, y de la mano del concepto de paradigma, la incomunicación entre las comunidades parece ser el paso que irremediablemente se tiene que dar.

\section{El lenguaje como determinante de la per- cepción del mundo \\ - El mundo a través del idioma materno}

Definir el concepto de comunidad, tanto en la teoría de Herder como en la de Kuhn, resulta esencial en tanto ése es el concepto fundamental del cual se deducen los posteriores. Si bien ya fue señalada la estrecha relación que establece Herder entre el Volkgeist como espíritu y sostén de la comunidad y el idioma, es sólo remitiéndonos a la teoría del lenguaje del alemán que podremos encontrar las bases para nuevas precisiones.

Herder establece, en primer lugar, una estrecha relación entre lenguaje y pensamiento ${ }^{6}$ para afirmar que sin lenguaje no hay pensamiento. Esto significa que pensamos a través del lenguaje $y$, por lo tanto, nos encontramos limitados por él. De esto se sigue que si cada comunidad se identifica por su idioma en tanto manifestación de su espíritu, y el idioma determina nuestra forma de pensar, cada comunidad piensa de diferente manera.

Como se desarrollará más adelante al tratar la teoría de Kuhn, no se trata simplemente de diferentes perspectivas que le pueden dar a los hechos las diferentes comunidades. Estamos hablando aquí de que cada comunidad percibe mundos diferentes y no meras interpretaciones sobre los mismos datos objetivos. Un seguidor de estas ideas fue otro alemán, Wilhelm von Humboldt (1767-1835), quien precisó y profundizó esta teoría herderiana. Según von Humboldt "la verdadera importancia del estudio del lenguaje radica en la participación de éste en la formación de representaciones." (Schaff, 1967:24)

Extremando aún más el vínculo entre lenguaje y nación, von Humboldt afirma la completa identidad entre ambos conceptos:

"El patrimonio intelectual y la forma lingüística de un pueblo se hallan tan íntimamente entreverados que si se diera uno de ellos, el otro se podría deducir completamente de aquél. El lenguaje es al mismo tiempo el fenómeno externo del espíritu de lo pueblos. El lenguaje es el espíritu y el espíritu es su lenguaje. No se les (distingue) nunca bastante." (Schaff, 1967:26)

Retomando la reconstrucción realizada por Adam Schaff (1967), la teoría del lenguaje de von Humboldt puede resumirse de la siguiente manera: Por un lado, el lenguaje media entre los seres que lo utilizan y las cosas a las que refiere; a su vez, en lo que se conoce como la tesis de la "forma interna", el lenguaje configura nuestra percepción del mundo de lo cual se sigue que a diferentes lenguajes le corresponden diferentes mundos. Por último, el espíritu nacional y la lengua funcionan como factores indiscernibles.

\section{- El mundo a través del paradigma}

En el último apartado sobre la teoría de Kuhn se había indicado que aquello que compartían los miembros de una comunidad científica era un paradigma. 
Este concepto es, tal vez, el más importante y aquel con el que Kuhn tuvo más dificultades. Especialmente a partir de un estudio de Masterman (1975) quien descubrió veintiún acepciones diferentes del término en la edición de 1962 de ERC, Kuhn se propone aclarar este punto en la "Posdata" a la edición de 1969. Allí Kuhn define al paradigma en dos sentidos: Como matriz disciplinar y como ejemplar. Sin embargo quiero detenerme en la versión que ya se encontraba en la edición de 1962 y que, probablemente con cierta ambigüedad, definía al paradigma como "una manera de ver el mundo". Esto no es más que afirmar que los miembros de paradigmas diferentes viven en mundos diferentes, esto es, perciben la realidad de modos diversos. Esta aseveración no tiene nada de metafórica: apoyado en los numerosos estudios de la teoría psicológica de la gestalt, Kuhn afirma que la percepción es mucho más que la información que reciben nuestras retinas desde el mundo. Lejos de haber una única realidad, ésta es una construcción humana donde intervienen, además de los datos que recibimos del "afuera", la historia, el idioma y los valores de la comunidad en la que estamos históricamente anclados.

Un elemento que puede ayudar a comprender esta cuestión está vinculado al significado del verbo ver. El propio Kuhn afirma haber utilizado adrede el doble sentido de ver (Kuhn, 1995b (1987): 97) que se hace inteligible tras su elucidación etimológica. Ver tiene, por un lado, un sentido visual o perceptivo por el cual puede entenderse como la manera de percibir a través de los ojos. Sin embargo, por otro lado, ver también tiene un sentido conceptual que significa comprender, entender y conocer. En las dos ediciones de ERC, Kuhn utiliza este doble sentido aclarando que el cambio conceptual conlleva un cambio perceptivo. Al establecer esta relación entre cambio conceptual y cambio perceptivo, Kuhn no está diciendo que ante un dato objetivo diferentes individuos interpretan cosas distintas. Esto implicaría que los datos son siempre los mismos, que se encuentren allí esperando nuestra interpretación. Al igual que en Herder, la apuesta de Kuhn es más fuerte: Es un objeto diferente el que se capta dado que la percepción es deudora de las estructuras conceptuales de los sujetos percipientes.

Pero es especialmente a partir de sus escritos de los años 80 que Kuhn entiende que no le ha dado la suficiente importancia al factor "lenguaje" en lo que respecta a la constitución del conocimiento.

Este giro hacia el lenguaje implica que el paradigma sea definido como un conjunto de categorías taxonómicas, un léxico compartido por un grupo de científicos. Éstos, entonces, comparten un conjunto de conceptos o términos y la referencia de los mismos. (Kuhn, 1995b (1987): 132)

Kuhn fue influenciado por la obra del filósofo analítico W.V.O. Quine y su tematización de la problemática de la traducción, como así también por el "segundo" Wittgenstein ". A su vez, esta nueva tesis de Kuhn también es deudora de los estudios de la Etnolinguística florecientes en la primera mitad del S. XX cuyo espíritu es claramente "neohumboldtiano". Es aquí donde se encuentra lo que se conoce como la hipótesis Sapir-Whorf, esto es, la tesis que afirma que la lengua no sólo refleja el pensamiento del hablante sino que también determina la manera en que éstos perciben el mundo. Esta hipótesis surgió de una serie de estudios sobre comunidades indígenas indoamericanas sin contacto con el resto de las civilizaciones. El caso paradigmático fue el de los hopi, tribu indoamericana que habitaba el territorio conocido como Arizona y en la cual Whorf puso particular atención.

El hecho de tratarse de una cultura indoamericana es clave porque se trata de una lengua que no es indoeuropea, es decir, una lengua que no deriva ni del griego ni del latín.

Las lenguas con un mismo origen incluso tan remoto como el griego y el latín, aun con todas las deformaciones y derivaciones, mantienen una misma manera de clasificar el mundo. En otras palabras, podremos decir "mesa" o "table" pero en ambos casos tenemos un término que designa un estado de cosas similar. De aquí que entre los idiomas de un mismo origen las dificultades de traducción sean menores dado que se mantiene vigente la misma estructura término-referente empírico.

Las dificultades aparecen cuando nos enfrentamos a un idioma con otro origen, siendo éste el caso de los hopi. Al estudiar esta tribu, Whorf cae en la cuenta que los hopi tienen una manera diferente de concebir el espacio y el tiempo.

Las categorías aparentemente intuitivas del espacio y el tiempo según la cosmovisión, llamemos, "occidental", son vistas como dos elementos analíticamente separables. No es éste el caso de la lengua hopi.

"Dos acontecimientos del pasado ocurrieron hace mucho tiempo -la lengua hopi no tiene ninguna palabra equivalente a nuestro "tiempo" - cuando entre ellos han ocurrido muchos movimientos físicos en forma tal que se haya recorrido mucha distancia ( ... ). La metafísica hopi no se plantea la cuestión de si la cosas que hay en un pueblo distante existen al mismo tiempo que las cosas que hay en el propio pueblo ya que ( ... ) dice que cualquier acontecimiento de un pueblo distante sólo puede ser comparado con otro acontecimiento en el propio pueblo mediante un intervalo de magnitud que contenga ambas formas, espacio y tiempo. Los acontecimientos ocurridos a distancia del observador sólo pueden ser conocidos objetivamente cuando han "pasado" (o sea cuando han entrado en el reino de lo objetivo) y cuanto mayor sea la distancia, mayor tendrá que ser el pasado ( ... ). Lo que ocurre en un pueblo distante ( ... ) sólo puede conocerse "aquí" más tarde. Si no ocurre "en este lugar", no ocurre tampoco en este tiempo; ocurre en "aquel" lugar y en "aquel" tiempo. Tanto el acontecimiento de "aquí" como el de "allí" se encuentran en el reino objetivo que en general corresponde a nuestro pasado, pero el acontecimiento de "allí" es el más lejano de lo objetivo, queriendo significar esto, desde nuestro punto de vista, que está mucho más lejos en el pasado, como también lo está en el espa- 
cio, que el acontecimiento de "aquí." " (Whorf, 1971) Esta sorprendente y hasta probablemente mucho más intuitiva categorización del espacio y el tiempo brinda un ejemplo claro a partir del cual difícilmente podamos sostener que un occidental globalizado y un hopi observan el mismo mundo.

Siguiendo con los hopi, podemos retomar el caso de la conceptualización que ellos realizan de los sustantivos y los verbos. En la lengua hopi cambia el criterio por el cual se clasifican los acontecimientos del mundo. Allí, los fenómenos se clasifican según su duración, de lo cual se sigue que el mundo se dividirá entre los acontecimientos más breves como una ola, un suspiro o una bocanada de humo, y los acontecimientos más duraderos como el dormir o el entablar una charla. Para los más breves se reservan los verbos y para los más duraderos, los sustantivos. Un ejemplo más cercano en el tiempo pero que viene a reflotar la hipótesis de Whorf acerca del relativismo lingüístico es el de los piraha, una pequeña tribu del Brasil que habita en la frontera con Bolivia. El caso, publicado en Science, , producto de la investigación de Peter Gordon de la Universidad de Columbia, Estados Unidos, y que cobró notoriedad en los diarios en 2004, resulta interesante particularmente porque los piraha tienen el lenguaje más pobre de los que se conocen -sólo tres vocales y ocho consonantes para los hombres y una menos (SIC) para las mujeresEl idioma de los piraha sólo tiene palabras para numerar el uno y el dos. Todo lo que sea más de dos se dice "varios": Tanto tres como 10658 manzanas serían "varias manzanas".

Siguiendo la tesis de Whorf, Gordon concluyó que la idea de número es ininteligible para los piraha dado que carecen de las palabras que permitan distinguir cantidades.

Pero con estos mismos datos, el antropólogo Dan Everett $^{10}$, nos acerca más a algunos de los puntos que venimos desarrollando.

"Tanto el lenguaje como el pensamiento de los miembros de esta tribu están relacionados con una forma de ver el mundo, una cultura. Es más, para Everett los pirahã no tienen ninguna forma de numeración, ni siquiera lo que se traduce como "uno, dos, varios" ; en realidad, ni siquiera diferencian masa de cantidad. El concepto de cantidad es para ellos esquivo y distinto del nuestro; ni siquiera existe una manera de comparar cantidades, porque la categoría "más que" esta ausente en su idioma." (Piscitelli, 7/ $11 / 2004)$

Es aquí donde se manifiesta claramente el cruce entre el idioma como manifestación del Volkgeist en Herder y seguido por von Humboldt, con los paradigmas kuhnianos entendidos como categorías taxonómicas. Es el lenguaje, sus términos, los que poseen una referencia determinada y a través del cual captamos el mundo. El mundo que se nos presenta, lejos de ser un único mundo asequible a la humanidad universalmente, es aquel que el lenguaje nos "permite" ver.

La gran incógnita que nos plantea esta tesis se relaciona con el problema de cómo podrán comunicarse estas comunidades que habitan mundos diferentes. Esto es lo que se desarrollará a continuación como el problema de la inconmensurabilidad.

\section{La Inconmensurabilidad \\ - La comunicación entre culturas incomparables}

En los apartados anteriores se desarrolló la idea de paradigma como una manera de ver el mundo, definición que en los últimos escritos de Kuhn fue sustituida por la de paradigma como léxico o categorías taxonómicas. Asimismo se había indicado el carácter exclusivo del paradigma en el sentido de su incapacidad de convivir junto a otro, aspecto que, en los términos de la teoría política de Herder, aparecía como el carácter monádico o cerrado de las comunidades. Ahora bien, la gran dificultad que tiene especialmente el discurso científico, aunque también el discurso político-sociológico, se vincula a la concepción de progreso. Si bien volveré sobre este punto más adelante, cabe adelantar que para poder hablar de progreso es necesario erigir patrones que permitan comparar en función de una determinada jerarquía qué elementos implican una evolución respecto de otros. El conflicto se plantea, tanto en Kuhn como en Herder, dado que según ellos, las comunidades científicas y políticas respectivamente son inconmensurables, esto es, incomparables.

Según Kuhn, una manera ilustrativa de acercarse a esta noción de inconmensurabilidad es a través de una analogía con la relación entre dos idiomas. El vivir en mundos diferentes, entonces, se asemeja a la situación en la cual dos hablantes con idiomas diferentes se enfrentan y son incapaces de comprenderse. ${ }^{12}$ En el caso de Herder, también se habla de inconmensurabilidad entre las comunidades y sus respectivas formas de ver el mundo. Lo que en Kuhn podría ser la negación de algún elemento supraparadigmático en lo que respecta a un método capaz de evaluar cada uno de los paradigmas de manera objetiva, en Herder puede interpretarse en clave de uno de los tópicos más en boga en estos tiempos, esto es, la crítica al etnocentrismo.

“Ideas que habíamos tenido por los axiomas más fundamentales de la razón humana, van desapareciendo aquí y allá con el clima de un lugar distante tal como la tierra firme desaparece a los ojos del navegante cual nube en el horizonte. Lo que el pueblo juzga parte imprescindible de su acervo conceptual, otro no lo ha pensado jamás, y hasta acaso lo tenga como perjudicial." (Herder, 1959: 233) Herder desarrolla muchas de sus ideas en el particular contexto alemán de fines del siglo XVIII, esto es, ante la amenaza militar y cultural de Francia. Es en ese contexto que Herder ve en lo intentos iluministas -particularmente franceses- un claro signo de etnocentrismo, de imposición de valores, contra el cual el pueblo alemán debe alzarse.

Para Herder, cada comunidad y cada tiempo histórico poseen sus propias normas y cualquier evaluación debe hacerse desde esas normas.

“Nada resulta más odioso para Herder que los abusos 
frecuentes de la comparación convertida en dueña y señora en un tribunal en que se censuran las expresiones poéticas y los resentimientos de un pueblo, de una época, de acuerdo con "las reglas del gusto" de otro tiempo." (Olender, 2005:58)

Lo único, la comunidad, es idéntica a sí misma y es incomparable en tanto no existe una escala de valores universal desde el cual poder realizar un juicio. Las filosofías de lo universal sólo encubren un pensamiento de lo particular con pretensiones imperialistas. Aparece en Herder, entonces, una revalorización de lo auténtico en el sentido de ser lo propio de cada comunidad más allá de que sus prácticas puedan resultar salvajes para un observador externo. Aquello que interesa es la originalidad y aquello a lo cual se teme es a las distorsiones a las que pueden llevar las intervenciones foráneas.

Sin embargo, paradójicamente, Herder, es un gran estudioso de las culturas a las cuales dedicó la mayor parte de su libro Ideas para una Filosofía de la Historia de la humanidad. Para Herder, las ciencias históricas, a diferencia de las naturales, deben apuntar a lo particular y concreto dado que es imposible encontrar leyes naturales que rijan de igual manera a las diferentes comunidades. Según Herder, si bien la traducción de la cosmovisión de una comunidad a otra es imposible, se puede realizar un ejercicio empático que permita, introduciéndose en el contexto a estudiar y asumiendo el lugar del otro, hallar cierto nivel de comprensión.

"Pues explicar las experiencias o actitudes humanas es ser capaz de trasladarse uno mismo mediante la imaginación empática a la situación de los seres humanos que se pretende estudiar; y esto equivale a comprender y comunicar la coherencia de una forma de vida concreta, de sentimiento y de actuación, y en consecuencia, la validez de un acto o acción dados, el papel que juegan en la vida y la perspectiva que sería "natural" en esa situación." (Berlin, 2000:201) (...) "No basta con entender las escrituras hebreas -nos dice Herder-, tampoco basta con comprenderlas como una sublime obra de arte y comparar su belleza con las obras de Homero (...) Debemos transportarnos a una tierra lejana y a una época remota, y leerlas como el poema nacional de los judíos, un pueblo de pastores y agricultores, escritas en lenguaje antiguo, sencillo, rústico y poético, no como texto filosófico o abstracto. (Berlin, 2000:238)

Asimismo, en su rotundo rechazo por las jerarquías, Herder también dedica parte de sus principales obras a tematizar otro tópico de la época, esto es, el de los "pueblos elegidos". Tanto en su versión religiosa como en su contrapartida laica, la historia de la humanidad fue y es testigo de pueblos o comunidades que se sienten "elegidas" y en un estadio superior sea de civilización, sea de acercamiento a la verdad. La coherencia de Herder se manifiesta en este punto en la negación de cualquier Favoritvolk (pueblo elegido): "La naturaleza, además, no hace que unas naciones sean intrínsecamente superiores a otras. Cualesquiera que fueran las cualidades de los antiguos germanos para considerarlos como el pueblo elegido por dios, creer que por ello, en virtud de sus cualidades creativas, poseerían el derecho a tener en sus manos al mundo entero y a dominar a otros pueblos, supondría una innoble vanidad de bárbaros. No hay ningún Favoritvolk." (Berlin, 2000: 211)

\section{- Intérpretes, traductores y bilingües}

Es claro que la inconmensurabilidad en su expresión más radicalizada, en el sentido de ausencia total de comprensión, lleva a un relativismo difícilmente justificable. Autores como Feyerabend ${ }^{14}$ no se preocupan por estas consecuencias pero éste no es el caso de Kuhn. De aquí que a lo largo de todos sus escritos Kuhn planteara, casi con obsesión, las razones por las cuales él no debía ser considerado un relativista. ${ }^{15}$

En este sentido ofrece algunos cambios a su tesis más radicalizada de la inconmensurabilidad.

Siempre pensado en términos de traducción, Kuhn comienza a hablar de la posibilidad de intérpretes e incluso de traductores. Un traductor es alguien que sabe dos idiomas y tiene la capacidad de trasladar los términos de un idioma al otro. No es éste el caso del intérprete quien frecuentemente sólo sabe un idioma y lo que hace es tratar de interpretar, desde su propia lengua o cosmovisión, una cultura o un idioma ajeno. Según Kuhn, el ser un buen intérprete es la condición de posibilidad para llegar a la traducción pero quien interpreta no necesariamente traduce. "La persona que interpreta busca ese sentido, se esfuerza por inventar hipótesis que hagan inteligible la inscripción, como por ejemplo que "gavagai" significa: "M irad, un conejo". Si tiene éxito lo que él ha hecho ( ... ) es aprender una lengua nueva, quizás la lengua en la que "gavagai" es un término, o quizás una versión más antigua de su propia lengua, una en la que los términos más usuales ( ... ) funcionaban de manera diferente. Si esta lengua puede traducirse a aquella con la que él comenzó es una cuestión discutible. A prender una nueva lengua no es lo mismo que traducir de ella a la propia. Tener éxito en lo primero no implica necesariamente que también se vaya a obtener éxito en lo segundo." (Kuhn, 1987b:105) La consecuencia de esta manera de enfocar el problema es que parece abrirse el camino hacia alguna posibilidad de comprensión al menos básica entre los paradigmas de las comunidades. Incluso siendo imposible la traducción, el intérprete podría, más allá de que su actividad es tan creativa -en el sentido de agregar sentidos- como ineficaz -en el sentido de que no puede captar con entera precisión todos los sentidos que lo estudiado posee en esa comunidad-, comprender el paradigma ajeno.

Pero Kuhn también se preocupa por su definición de inconmensurabilidad dado que este concepto llevado al extremo le impediría salir del relativismo, la irracionalidad y la incomprensión.

De aquí que comience a hablar de inconmensurabilidad local, en el sentido de que existen términos que de paradigma en paradigma mantienen su referencia, siendo la inconmensurabilidad un atributo solamente de algunos términos.

Claramente, y más allá de las dificultades que puedan 
surgir respecto a la coherencia interna de la teoría de Kuhn, queda claro que la inconmensurabilidad local es un intento de hacerse a un lado de las acusaciones de relativismo e irracionalismo que Kuhn recibió después de la publicación de ERC. Sin embargo, Kuhn da un nuevo giro a esta tesis en uno de sus últimos escritos (Kuhn, 1990). Allí introduce el término " bilingüismo" para tratar de retratar esta "nueva" idea de la posibilidad de comprensión de un paradigma. Sin embargo, a mi juicio, esta nueva noción lo acerca a Kuhn a una versión más radicalizada. La traducción de los términos de un paradigma a otro será llevada a cabo por un bilingüe, esto es, un sujeto que comprenda los dos idiomas. Sin embargo, a diferencia del traductor, el bilingüe tiene la capacidad de comprender los léxicos de cada uno de los paradigmas en sí mismos pero es incapaz de trasladar los términos de uno a las categorías del otro. En otras palabras, el bilingüe no traduce: Sólo tiene la capacidad de comprender dos paradigmas dentro de sus estructuras. Esta última versión, semejante al esfuerzo empático propuesto por Herder, si bien permite cierto nivel de comprensión, traerá grandes dificultades para tematizar una noción tan intuitiva como lo es la del Progreso.

\section{Las dificultades del progreso}

- Filosofía de la historia y metas intracomunitarias Quien considere que la cuestión del progreso fue y es sólo una discusión propia del campo de la ciencia no está observando la magnitud de la problemática. En general, podemos decir que el tópico del progreso tal como llega hasta nuestros días es producto de las discusiones del S. XVIII. Allí, los autoproclamados "modernos", término que no tenía nada de neutral, buscaban diferenciarse de los antiguos no sólo en tanto diversos sino en tanto superiores.

La etimología del término progreso señala que como participio de "progredi" y proveniente del latín progressus, éste debe entenderse como un "ir hacia adelante o hacia mejor." Claramente, esta definición muestra los compromisos que la vinculan a una doctrina teleológica, esto es, una concepción que supone la existencia de una determinada finalidad, sea ésta un compromiso con la divinidad, alcanzar la felicidad o ser el mejor jugador de fútbol de todos los tiempos. Esta finalidad hace las veces de referencia y es ésta la que posibilita evaluar en qué sentido se está o no progresando. A manera de ejemplo, un estudiante universitario que tiene como referencia el conseguir su título de Profesor o Licenciado -treinta materiaspodrá determinar que veinte materias aprobadas implica un progreso comparado con la situación en la que estaba cuando había aprobado sólo diez. Ahora bien, ni Herder ni Kuhn suponen que existe alguna finalidad que esté por encima de las comunidades sociales y científicas respectivamente, es decir, no existe referencia supracomunitaria que permita establecer metas y, por lo tanto, progreso.

En el caso de Kuhn, no existe una finalidad que trascienda a los paradigmas sino que cada paradigma establece su fin que es, como quedó desarrollado anteriormente, inconmensurable respecto al resto. En el caso de Herder, la diversidad es parte del plan divino y los fines son atribución de las comunidades. Aquí creo necesario hacer una pequeña digresión para hacer hincapié en las razones por las cuales considero preciso tematizar el progreso no sólo en el ámbito científico. Como se indicó en el comienzo del apartado, la problemática del progreso parece haber sido el producto del contexto especial del S. XVIII. Ese enfoque de carácter general en el sentido de desarrollarse no sólo en el ámbito de las ciencias sino también en el terreno de lo moral, parece estar languideciendo desde la segunda mitad del S. XX. Los avances contra la discriminación racial y cultural parecen ser claros síntomas de estos cambios. Sin embargo, aunque solapadamente, la discusión acerca de criterios para determinar "mejores y peores" 0 "lo bueno y lo malo" se encuentra en el fondo de las cuestiones más dramáticas que aquejan al mundo. Toda la problemática filosófica acerca de la intervención en países ajenos, lleva de fondo una implica que, por ejemplo, el respeto por los derechos humanos se presenten como un progreso respecto de la situación anterior, de lo cual se sigue que, en caso de que exista una comunidad o país que no los respete, podría llegar, incluso, a ser invadido en nombre de la civilización.

En muchos círculos, la disputa Oriente y Occidente suele expresarse en términos de Civilización vs. Barbarie. Es por eso que la cuestión acerca del progreso me parece esencial en el debate político también. De hecho, en otro contexto, pero con dificultades similares, Herder intentará desestimar la idea de una finalidad o un ideal de las comunidades dentro del cual se las puede evaluar. Su nacionalismo y su lucha contra el etnocentrismo -ejemplificado en la figura de los franceses-, son análogos a muchas de las posturas de los grupos que resaltan su particularidad, casi siempre, en oposición a las políticas de Estados Unidos hoy en día.

Retomando la tesis de Herder acerca del progreso, se puede afirmar que existen dos interpretaciones antagónicas -tal vez igualmente plausibles- de la misma, si bien a continuación sólo se tendrá en cuenta aquella coherente con las tesis del autor desarrolladas anteriormente.

Parece claro que del realzamiento de lo particular, y la inconmensurabilidad de las comunidades se sigue la imposibilidad de trazar una línea de progreso en la historia de la humanidad. En este terreno, los desarrollos de Herder son muy ricos dado que sus grandes obras tuvieron como tópico el problema del progreso ${ }^{16}$. Con la misma carga metafísica que Kant y Hegel aunque en clave teológica, Herder, afirma que el plan de la providencia que rige la historia determina que ningún momento histórico y ninguna comunidad es superior a otra. No existe el pueblo elegido ni se puede trazar una jerarquía de civilizaciones. Cada una de las civilizaciones es un fin en sí puesto por Dios como parte de un plan que es inaccesible a los humanos. "La coordinación de este movimiento cíclico en que las culturas se siguen unas a otras pero no se ase- 
mejan, la gestión de esta dramaturgia grandiosa, está signada por el destino que llama a cada nación (...) cada una a su hora. De tal modo, la historia se desenvuelve en olas sucesivas (...) cuya significación total escapa necesariamente (...) al género humano." (Olender, 2005:61)

Al etnocentrismo de los pensadores de los "pueblos superiores", Herder opone la autarquía de las civilizaciones. Dado que cada comunidad tiene su propia finalidad, el progreso será interno a la comunidad y evaluable sólo desde adentro. Consciente de la importante carga que conlleva consigo el término progreso, Herder reemplaza la palabra alemana Verbesserung -progreso como "signo de perfeccionamiento" - por el término Fortgang -progreso como "innovación permanente".

De esta manera y bajo esta interpretación, Herder parece distanciarse de las filosofías de la historia de Kant y Hegel. En estos autores parece más claro que existe una finalidad que trasciende las comunidades particulares. En el caso de Kant, el secreto designio de la naturaleza nos lleva -si bien como ideal regulativo- a la Confederación de Estados mientras que en Hegel, la astucia de la razón lleva a la Idea a concretizarse, en su punto cúlmine, en el Estado Prusiano de las primeras décadas del S. XIX. Sin embargo, no se puede dejar de soslayo la otra interpretación a la que da lugar Herder. Me refiero a los pasajes donde da explícita primacía a los pueblos nórdicos, estableciendo una jerarquía entre naciones en pos de la meta máxima de la humanidad, esto es, el cristianismo de Jesús.

“(Herder) pasa así de una visión generosa en que todos los pueblos tienen iguales méritos, unos y otros capaces de una felicidad tan incomparable e incomunicable como absoluta, a una escala de valores en que los blancos, cristianos de Europa, son los únicos privilegiados. Sus ventajas, determinadas por una geografía clemente y un clima templado, son la expresión de una elección divina." (Olender, 2005:63)

\section{- Progreso con y sin referencias}

Al igual que Herder, Kuhn se enfrenta a la dificultad, sin negar el progreso científico, de mantener la tesis de la inconmensurabilidad entre paradigmas.

La primera respuesta de Kuhn es similar a la que daría Herder: El progreso sólo existe dentro del paradigma porque es la comunidad científica que sostiene el paradigma la que determina la finalidad del mismo. Según Kuhn, el progreso científico se da dentro de lo que él llama Ciencia normal, esto es, el momento histórico en el que un paradigma ha prevalecido sobre otros y donde en función de constantes intentos de adaptación a lo real va resolviendo los enigmas de la naturaleza. El gran problema que se le plantea a Kuhn es cómo justificar el progreso entre paradigmas, es decir, cómo poder afirmar que un paradigma es mejor que otro si, al fin de cuentas, éstos son inconmensurables. Aquí, la teoría de Kuhn entra en tensión: Según éste, en los momentos donde se produce una revolución científica, esto es, en la circunstancia donde un paradigma es reempla- zado por otro, también se da un progreso científico. Más allá de la incomunicabilidad entre paradigmas, el sólo hecho del reemplazo de uno por otro permite decir que el reemplazante implica un progreso respecto de su reemplazado. Coexisten, entonces, en Kuhn, dos maneras de entender el progreso: Una intraparadigmática que es caracterizada por un progreso de carácter lineal y acumulativo y una interparadigmática donde el progreso se da a través de rupturas y de manera discontinua.

Una vez más, la inconmensurabilidad parece ser una tesis difícil de sortear sin caer en contradicciones a la hora de afirmar un progreso a lo largo de la historia.

\section{...y sin embargo nos comunicamos}

Las dos posturas relativistas desarrolladas a lo largo de este trabajo muestran por un lado muchísimas similitudes. Más allá de ello, interesa rescatar las derivaciones que estas teorías tienen a la hora de plantear la problemática de la comunicación.

Por un lado, encontramos que uno de los principios fundamentales de este relativismo es la tematización de la comunidad como cerrada y determinante del individuo. En el ámbito social, las comunidades cerradas o las nacionalidades homogéneas podían tener un correlato empírico hasta el siglo XX donde las grandes migraciones asociadas a las hambrunas y a las guerras, conformaron un escenario multicultural novedoso. Si bien no podemos exigirle a Herder una visión de futuro tal, no es menos verdad que existe en la actualidad una corriente importante de nuevos pensadores que parecen describir el nuevo escenario con las anacrónicas categorías del filósofo romántico del S. XVIII.

Salvo contadas excepciones, los Estados albergan diversas culturas que, en algunos casos con dificultades, conviven y modifican su tradición. De esta manera, este ida y vuelta que surge de la conexión de las civilizaciones algunas de las cuales ni siquiera son coetáneas geográficamente, derriba el mito metafísico de un Volkgeist impermeable a la interacción con otras culturas y con otras lenguas.

En el plano de la ciencia, la construcción de la comunidad científica en función del compartir un paradigma, se diferencia de la postura herderiana en cuanto a los nulos compromisos kuhnianos con una justificación metafísica de la comunidad. ${ }^{18}$ Sin embargo, el vínculo comunitario de los científicos parece tan fuerte como el descrito por Herder.

Pero una vez más, la tesis de Kuhn parece ser demasiado tajante si se piensa que no existe contacto con un "afuera" o con otras "manera de ver el mundo". Tal vez, una forma de salvar el escollo, podría ser interpretar la noción de paradigma de manera más laxa para ampliar su alcance espacio-temporal. De esta manera la noción de paradigma podría incluir los diferentes intercambios que se pueden dar a lo largo de la vigencia del paradigma haciendo que éste padezca grandes transformaciones a lo largo de su vida útil.

También es preciso reconocer la rigurosidad de la descripción de Kuhn en cuanto al funcionamiento 
de las estructuras académicas y su carácter exclusivista. Sin embargo, deberíamos pensar hasta qué punto el nuevo incesante flujo de información hace mucho más difícil pensar en comunidades homogéneas y aisladas.

En cuanto al segundo punto desarrollado, esto es, la forma en que el lenguaje determina nuestra percepción del mundo, la discusión no parece tener fin y asistimos a continuas "reinvenciones" de posturas. Desde el debate entre Platón y los sofistas acerca de la naturaleza del lenguaje hasta el enfrentamiento entre el universalismo de Chomsky y las tesis relativistas de los etnolingüistas, se trata de la misma discusión sólo que en contextos históricos diferentes. Tal como se presentan, parece haber buenas razones para apoyar a cada una de ellas. Por ello es que propuse tratar la cuestión en el terreno de la inconmensurabilidad y el problema de la traducción. En ese terreno, mi postura puede sintetizarse en el siguiente factum: "...y sin embargo nos comunicamos". En otras palabras, si bien el relativismo nos brinda numerosos ejemplos de teorías o visiones aparentemente inconmensurables, no nos explica la razón por la cual, al fin de cuentas y con mayor 0 menor precisión, logramos comprender al otro. En todo caso, la renuncia a la traducción radical, no nos lleva necesariamente a la incomunicación de lo cual se sigue que, si bien la traducción total se presenta como una utopía, puede servirnos como ideal regulativo en nuestro afán de comprender.

Por último e íntimamente vinculado al concepto de inconmensurabilidad, la problemática del progreso parece ser uno de los puntos más álgidos de la discusión. En este sentido podemos señalar una vez más, los innumerables intentos que tanto Kuhn como Herder llevaron adelante para no caer en la contraintuitiva y difícilmente sostenible idea de la imposibilidad de afirmar el progreso. En este punto, debemos distinguir el ámbito de la ciencia del de lo social. Más allá de las simpáticas posturas posmodernas que mostrando la estrecha relación entre ciencia e historia derivan de ello la ausencia de

\footnotetext{
Notas

${ }^{1}$ Ver Huntington, S., (2000) El choque de civilizaciones. Buenos Aires: Paidos.

2 Para ser más precisos, en la teoría de Herder esta noción convive con una postura antagónica, esto es, la que establece jerarquías de naciones en función de su carácter más o menos civilizado. Volveré sobre este punto en el último apartado de este trabajo.

${ }^{3}$ La misma argumentación utiliza uno de los principales pensadores de la filosofía política de la actualidad, Will Kymlicka, a la hora de defender la asignación de derechos colectivos a determinados grupos/culturas. Ver Kymlicka, W., (1996), Ciudadanía Multicultural, España, Paidos.

${ }^{4}$ Reichenbach, H., (1938), Experience and prediction. Chicago: University of Chicago Press.

${ }^{5}$ Es posible afirmar que en las disciplinas sociales la situación es diferente pero ello, lejos de ser visto de manera positiva, es señalado por Kuhn como el elemento que impide la madurez de estas disciplinas.

${ }^{6}$ Herder was one the first to insist on the close relation
}

cualquier fundamento o patrón objetivo para evaluar, considero difícilmente justificable la afirmación que indica que entre los comienzos del homo sapiens hasta hoy no ha habido progreso y que se trata simplemente de conocimientos "diferentes".

Pero en el plano social y particularmente en el terreno moral resulta más controvertido afirmar que ha existido un progreso. Sin duda el S. XX ha sido tal vez el siglo testigo de las peores masacres lo cual nos lleva a preguntarnos hasta qué punto se ha producido un avance moral y social. Sin embargo, también es verdad que, si bien queda mucho por hacer, la segunda mitad del S. XX ha puesto en la agenda de la humanidad el respeto por los derechos humanos como un ideal cuyo no respeto lleva cada día más a la presión de la opinión pública y de los Estados. Sin apoyarme en fundamentos metafísicos del Derecho natural, considero que la situación actual de conciencia acerca de la dignidad humana implica un paso adelante en la historia de la humanidad y aquellos que desde una versión pseudo-apocalíptica señalan las atrocidades que se siguen cometiendo parecen no tener en cuenta que la denuncia de esos hechos como tales sólo es posible a partir de la conciencia que la humanidad ha adquirido en las últimas seis décadas. Es por todo esto que considero que las posturas relativistas pueden ser útiles para poner en tela de juicio muchos presupuestos que acríticamente sostenemos pero no son capaces de describir correctamente el estado de cosas actual ni de brindar herramientas normativas que permitan resolver las circunstancias apremiantes que nos toca vivir. Puntualmente, aplicado a la temática que este artículo ha convocado, el relativismo debe llamarnos la atención y hacernos conscientes de que muchas veces nuestra actitud para con el mundo o para con otras culturas se encuentra sesgada por nuestra historia y nuestra identidad pero esto no significa que carezcamos de algún parámetro para evaluar, comunicarnos y comprender las acciones de los otros. between culture and language, and to argue that language was not just a means of communication and selfexpression but an embodiment and vehicle of culture. (Parekh, 2000:72)

Ver Quine, W. V. O., (1960), Word and object, Cambridge: MIT Press.

${ }^{8}$ Ver W ittgenstein, L., (2004), Investigaciones filosóficas, M éxico: Crítica.

${ }^{9}$ Gordon, P., Numerical Cognition Without Words:

Evidence from Amazonia, Science [publicado On-line 19/ 8/2004]

${ }^{10}$ Piscitelli, A., El relativismo lingüístico y el ejemplo de los piraha, http://www.ilhn.com/filosofitis/ [publicado el 7/11/ 2004]

${ }^{11}$ El término "inconmensurabilidad" surge de la conjunción del prefijo "in" (negación) con el término latín commetiri que significa "comparar". En sus orígenes, hacía referencia a una dificultad propia de las matemáticas relacionadas al cálculo de longitudes. De esta manera, 
había inconmensurabilidad cuando entre dos longitudes a y $b$ no era posible encontrar una unidad de longitud $u$, con la que pudieran medirse tanto a como b.

Según Fernández Moreno (1997) en la teoría de Kuhn podemos hablar de inconmensurabilidad en tres sentidos diferentes: un sentido semántico aplicado a la idea de que diferentes léxicos no son intertraducibles; un sentido ontológico, entendiendo por tal la idea de que los paradigmas son inconmensurables en tanto captan mundos diferentes y un sentido metodológico que indica que no existe método objetivo o racional capaz de evaluar a los diferentes paradigmas. Ver Palma, D. (2005)

${ }^{3}$ Algunos años después de la muerte de Herder, la amenaza de invasión militar francesa se efectivizó lo cual llevó a muchos filósofos y pensadores alemanes a retomar las banderas de Herder. Este es especialmente el caso de Fichte. Ver Fichte, J. G., (1984), Discursos a la nación alemana, Argentina: Ediciones Orbis.

${ }_{15}^{14}$ Ver Feyerabend (1984).

${ }^{15}$ Por citar sólo una de sus explícitas alusiones al tema: "Propiamente entendida -algo que no siempre he manejado bien- la inconmensurabilidad está lejos de constituir una amenaza para una evaluación racional de las pretensiones de verdad, tal como frecuentemente ha parecido". (Kuhn, 2002 [1990]: 8)

\section{Bibliografía}

- Berlin, I. (2000) Vico y Herder, Madrid: Cátedra.

- Feyerabend, P., (1984), Contra el método. Buenos Aires: Ediciones Orbis.

- Formigari, L., (2004) History of Language philosophies, Philadelphia. USA: J. Benjamins publishing Company.

- Gómez, R., (1993), Kuhn y la racionalidad científica. ¿Hacia una kantianismo postdarwiniano? En Nudler, O. y Klimovsky, G., La racionalidad en debate, Buenos Aires: C.E.A.L.

- Gordon, P., Numerical Cognition Without Words: Evi dence from Amazonia, Science, [publicado On-line 19/8/2004]

- Herder, J. G.

(1959) Ideas para una Filosofía de la Historia de la humanidad (traducción directa de J. Rovira Armengol), Bs. As.: Losada.

(1954) La idea de humanidad (Selección, traducción, prólogo y notas de Catalina Schirber), Bs. As: Fascículo 40 de la Antología alemana editada por el Instituto de Literatura alemana, FFyL, UBA.

(1950) Filosofía de la historia para la educación de la humanidad (Introducción de E. Pucciarelli), Bs. As.: Editorial Nova.

- Klimovsky, G. (1993) Inconmensurabilidad, incomunicación e irracionalidad, en Nudler, O. y Klimovsky, G., La racionalidad en debate, Buenos Aires: C.E.A.L.

\section{- Kuhn, T, S.}

(2002) [1990], El camino desde La Estructura, Phrónesis, Año 2, N. 6 .

(1995c) [1987], Racionalidad y elección de teorías en Kuhn, T. S., ¿Qué son las revoluciones científicas? y otros ensayos, España: Altaya.

(1995b) [1987], Conmensurabilidad, comparabilidad y comunicabilidad, en Kuhn, T. S., ¿Qué son las revoluciones científicas? y otros ensayos, España: Altaya. (1995a) [1987], ¿Qué son las revoluciones científicas?, en Kuhn, T. S., ¿Qué son las revoluciones científicas? y otros ensayos, España: Altaya.

(1993) [1962/1969], La estructura de las revoluciones científicas, M éxico: F.C.E.

(1985) La Revolución Copernicana, Madrid, Hyspamérica.
${ }^{16}$ Especialmente Otra filosofía de la historia de 1774 e Ideas para la filosofía de la historia de la humanidad de 1784-1791.

Creo que esto se puede ver claramente en la teoría de Darwin y en su mal interpretado concepto de evolución. Si bien Darwin nunca habló de progreso, podemos decir que la idea que está de fondo en su teoría se asemeja al concepto de Fortgang (sin carga metafísica, por supuesto) en el sentido de que no se trata de un progreso con miras a un fin. Justamente lo que Darwin muestra y genera mayor escándalo, es que las modificaciones específicas son completamente fortuitas de lo cual se sigue que las transformaciones no acercan a las nuevas especies a la especie ideal.

${ }^{18}$ Esto no impide, obviamente, que los miembros de la comunidad compartan principios metafísicos que se aplican a lo real pero no significa que la justificación de la unión de la comunidad responda a alguna inasible entidad.

${ }^{19}$ Por ejemplo, el paradigma aristotélico-tolemaico que estuvo vigente unos 2000 años a lo largo de todo el espectro, llamemos, "occidental". Incluso podríamos pensar en el paradigma copernicano, aquel que, si bien se mantuvo vigente diez veces menos que su antecesor, albergó una cantidad de cambios tal que invitan a la reflexión acerca de cuán limitante es un paradigma.

(1982), La tensión esencial. Estudios selectos sobre la tradición y el cambio en el ámbito de la ciencia, México: F.C.E. (1975), Consideraciones en torno a mis críticos, en Lakatos, I. y Musgrave,A., La crítica y el desarrollo del conocimiento, Barcelona: Grijalbo.

(1963), The function of dogma in scientific research, en Crombie, A., C., (comp.), Scientific change, Heinemann, pp. 347-365.

- Logos Multilingual portal, Elmundo creado por las palabras http://www.logos.it/pls/dictionary/linguistic_resources.cap 2_9?lang=es [Consulta:1/11/2004]

- Ōlender, M., (2005) Las lenguas del paraíso, Argentina: F.C.E.

- Padilla, E. Consideraciones acerca de la inconmensurabilidad, http://www.pucp.edu.pe/eventos/congresos/ filosofia/programa_general/jueves/sesion9-10.30/ PadillaElizabeth.pdf. [Consulta: 1/12/04]

- Palma, D. A. (2005) Relativismo e inconmensurabilidad. Apuntes sobre la filosofía de T. Kuhn, Bs. As.: Baudino ediciones.

- Palma, H. y Wolovelsky, E. (2001) Imágenes de la racionalidad científica, Buenos Aires: Eudeba.

- Parekh. B. (2000) Rethinking Multiculturalism: Cultural diversitiy and polithical theory. New York, USA: Palgrave Publishers.

- Pilling, M. y Davies, I. R. L. (2004) Linguistic relativism and colour cognition, British journal of Psychology, N. $\mathrm{o}$ 95, pp. 429-455.

- Piscitelli, A. El relativismo lingüístico y el ejemplo de los piraha, http://www.ilhn.com/filosofitis/ [publicado el 7/ 11/2004]

- Sankey, H.

(1998), Taxonomic incommensurability, Inter-national Studies in the Philosophy of Science, N. 12, pp. 7-16. (1991), Incommensuability, translation and understanding, Philosophical Quarterly, Vol. 41, Fascículo 165.

- Schaff, A., (1967) Lenguaje y conocimiento, México:Grijalbo.

- Whorf, B. L. (1971) Lenguaje, pensamiento y realidad, Barcelona, Seix Barral. Extraído de http://www.inicia.es/ de/diego_reina/filosofia/antropologia/whorf.htm [Consultà: 15/11/2004] 